HNO 2011 · 59:318-318

DOI 10.1007/s00106-011-2285-0

Online publiziert: 4. April 2011

(c) Springer-Verlag 2011

J. Schipper

Universitäts-HNO-Klinik Düsseldorf

\title{
Schädelbasischirurgie in Deutschland
}

\section{Vom Mikroskop zum Endoskop, von Radikalität zur Funktionalität}

te Chirurgie (CAS) bzw. Navigation einzusetzen. Mit dem zusätzlichen Einsatz von Endo-skopen lassen sich heute auch über kleinste Zugangswege Strukturen visuell erfassen, die zur Schädelbasis abgewinkelt verborgen liegen. Die verbesserte Präzision in der digitalen Bildgebung und im Neuromonitoring erlaubt eine optimierte Anpassung von Implantaten oder Neuroprothesen, beispielsweise für die Hörrehabilitation oder die Behandlung zentralnervöser Erkrankungen wie M. Parkinson.

\section{( $)$ Die verbesserte Präzision in der digitalen Bildgebung erlaubt eine optimierte Anpassung von Implantaten}

risierte Tumoren operativ entfernen.

Die verbesserte Auflösung von radiologischen Bildschichtverfahren wie in der Computertomographie (64-Zeiler-Multislice-CT) oder Magnetresonanztomographie (7,5-T-MRT) erlaubt präzise Aussagen über die Tiefen- und Nachbarinfiltration von Tumoren wie nie zuvor und damit eine verbesserte Operationsplanung einschließlich Aussagen über die Organfunktionalität. Durch ergänzenden intraoperativen Einsatz der digitalen Volumentomographie (DVT) können darüber hinaus während der Operation jederzeit digitale Bilddaten reevaluiert werden, um sie für eine präzise computerassistier-
Das Zusammenführen des fachübergreifenden Wissens, etwa aus den Bereichen Neuroradiologie, Neurochirurgie, MundKiefer-Gesichts-Chirurgie, Hals-NasenOhren-Heilkunde sowie Kopf-Hals-Chirurgie, Ophthalmologie und Strahlentherapie in Form interdisziplinärer Zentren ermöglicht es heutzutage, schädelbasisnah gelegene Tumoren funktionserhaltend und weitestgehend kosmetisch wenig sichtbar operativ zu entfernen und zu therapieren. In den nachfolgenden Arbeiten wird jeweils aus der Sicht der jeweiligen Fachdisziplinen aus Neuroradiolo- gie, Neurochirurgie, Mund-Kiefer-Gesichts-Chirurgie sowie Hals-Nasen-Ohren-Heilkunde und Kopf-Hals-Chirurgie hierzu ein aktueller Überblick gegeben.

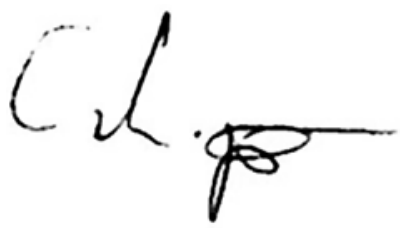

Prof. Dr. Dr. h.c. Jörg Schipper

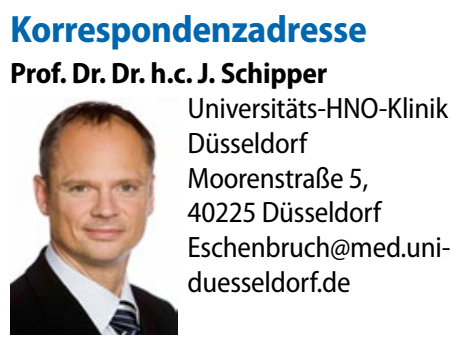

